\title{
Projections of Incidence, Prevalence and Mortality from Melanoma in Spain
}

\author{
Milagros Bernal $^{1 *}$, Dyego L. B. Souza ${ }^{2}$, German Gomez ${ }^{1}$, Francisco J. Gomez ${ }^{3}$ \\ ${ }^{1}$ Universidad de Zaragoza, Zaragoza, Spain; ${ }^{2}$ Universidad de Rio Grande, Port Alegre, Brazil; ${ }^{3}$ Atención Primaria de Salud, Zaragoza, \\ Spain. \\ Email: *milagrosbernalperez56@gmail.com
}

Received April 23 $3^{\text {rd }}, 2013$; revised May 22 ${ }^{\text {nd }}, 2013$; accepted May 29 ${ }^{\text {th }}, 2013$

Copyright (C) 2013 Milagros Bernal et al. This is an open access article distributed under the Creative Commons Attribution License, which permits unrestricted use, distribution, and reproduction in any medium, provided the original work is properly cited.

\begin{abstract}
Objective: The incidence and mortality rates for cutaneous melanoma (CM) have increased markedly in the last few years. As such, the aim of this study was to forecast their progression in Spain up until 2022. Design: A prospective epidemiological study using the Miamod technique. The primary data source was the mortality statistics released by the National Statistics Institute. Study selection: The MIAMOD method was used. Data extraction: Cases of death due to general and melanoma-related mortality in Spain between 1998 and 2007. Results: In men, the incidence is expected to increase from 11.85 (adjusted: 8.52) to 14.79 (adjusted: 9.43) per 100,000 inhabitants over the period 1998-2022. Similarly, the mortality is expected to increase slightly from 5.84 (adjusted: 4.12) to 7.19 (adjusted: 4.49) per 100,000 inhabitants, and the prevalence from 73.72 (adjusted: 52.31) to 95.46 (adjusted: 60.02) per 100,000 inhabitants over the same period. In women, the incidence is expected to increase from 3.79 (adjusted: 3.04) to 6.46 (adjusted: 4.21) per 100,000 inhabitants between 1998 and 2022, although this increase is not statistically significant. The mortality is expected to increase from 1.67 (adjusted: 1.24) to 2.98 (adjusted: 1.86) per 100,000 inhabitants, and the prevalence from 50.04 (adjusted: 44.54) to 84.81 per (adjusted: 56.18) 100,000 inhabitants over the same period. Conclusions: Primary and secondary prevention programs for this disease must be maintained.
\end{abstract}

Keywords: Projections Cutaneous Melanoma; Incidence; Mortality

\section{Introduction}

The incidence and mortality rates for cutaneous melanoma (CM) have increased markedly worldwide in the past few years, especially in Caucasian populations, with the highest rates being found in Australian men (66.4; adjusted rate: 50.7) [1]. Although the rates in Europe are lower, Spain is one of the countries in which, despite the fact that significant increases have been observed, the effects of preventive measures are expected to reduce this trend in the near future.

In this work, we have attempted to forecast the changes in melanoma incidence and mortality rates in Spain. This disease is responsible for some $0.7 \%$ of all deaths, with a mortality rate of 1.8 per 100,000 inhabitants [2]. The aim of this study was to estimate future trends in the incidence and mortality rates for this disease using new ad hoc statistical techniques and therefore to determine whether the preventive measures implemented to date have

${ }^{*}$ Corresponding author. proved effective.

\section{Materials and Methods}

All cases included belong to the "malignant melanoma of skin" item (C43) and were obtained from the National Statistics Institute (INE) [3] publication "Deaths according to cause of death" (ICD-10), which includes the mortality for this disease.

The histopathological verification percentage for the cases diagnosed is $100 \%$ as the clinical diagnosis of this severe disease is accompanied by a subsequent confirmatory diagnosis, which is always anatomopathological.

The MIAMOD program [4], which allows projections regarding non-transmissible diseases to be made, was used. This statistical software program allows future incidence, prevalence and mortality rates to be estimated. A back analysis, which involved recalculating the mortality data required to initiate the program in order to be able to correlate the observed and estimated data and 
thereby evaluate the validity and reliability of the estimates, was also performed.

The incidence was calculated using a Poisson regression, which provides highly reliable estimates of mortality. The model calculates the crude and standardised incidence rates per 100,000 inhabitants for the age distribution of the European population. The program used Weibull's parametric model [5], and the relative survival data were obtained from the melanoma item of Enrocare [6] for all Spanish registries.

The best model was selected on a likelihood basis. The model was validated by checking that the estimates obtained retrospectively were comparable to the observed mortality in Spain.

As well as the data for melanoma-related mortality, the mortality for any reason in Spain and the population structure between 1998 and 2007 were used by the program [7].

The values estimated using the MIAMOD program were used in a trends study using the Joinpoint regression program [8] in order to determine whether the estimated trends were statistically significant and their annual variation, as measured by the annual percentage change (APC).

\section{Results}

\section{Projections in Men}

The incidence is expected to increase from 11.85 (adjusted: 8.52 ) to 14.79 (adjusted: 9.43) per 100,000 inhabitants over the period 1998-2022.

Similarly, the mortality is expected to increase slightly from 5.84 (adjusted: 4.12) to 7.19 (adjusted: 4.49) per 100,000 inhabitants, and the prevalence from 73.72 (adjusted: 52.31) to 95.46 (adjusted: 60.02) per 100,000 inhabitants over the same period (Table 1, Figure 1).

The three-year survival, as calculated using the same program, is expected to improve from $0.93 \%$ to $0.94 \%$ for the $55-64$ years age group, from $0.85 \%$ to $0.86 \%$ for the $65-74$ years age group, and from $0.53 \%$ to $0.61 \%$ for the 75 - 99 years age group.

The Joinpoint application showed that the APC for the incidence in both men and women tends to increase, although not in a statistically significant manner, whereas the mortality in men increases significantly by $2.35 \%$ (p $<0.0001$ ) between 2002 and 2022 .

Projections in women: The incidence is expected to increase from 3.79 (adjusted: 3.04) to 6.46 (adjusted: 4.21) per 100,000 inhabitants between 1998 and 2022, although this increase is not statistically significant.

The mortality is expected to increase from 1.67 (adjusted: 1.24) to 2.98 (adjusted: 1.86) per 100,000 inhabitants, and the prevalence from 50.04 (adjusted: 44.54 ) to 84.81 per (adjusted: 56.18) 100,000 inhabitants over the same period (Table 2, Figure 2).
The Joinpoint application gave an APC of $-2.51 \%(\mathrm{p}$ $=0.65)$ for the incidence and $2.35 \%$ for the mortality $(\mathrm{p}=$ 0.002 ).

The three-year survival improves from $0.90 \%$ to $0.92 \%$ for the $65-74$ years age group and from $0.58 \%$ to $0.65 \%$ for the 75 - 99 years age group.

\section{Discussion}

The main cause of exposure is exposure to ultraviolet radiation [9], together with various predisposing factors such as genetic susceptibility [10] and other risk factors, including geographical distribution and individual solar protection. These geographical variations in risk factors suggest the need for continual monitoring in order to determine the evolution of melanoma incidence rates. Early diagnosis and improved treatment should result in lower mortality rates. Finally, software improvements will allow possible variations in a specific geographic region to be estimated appropriately.

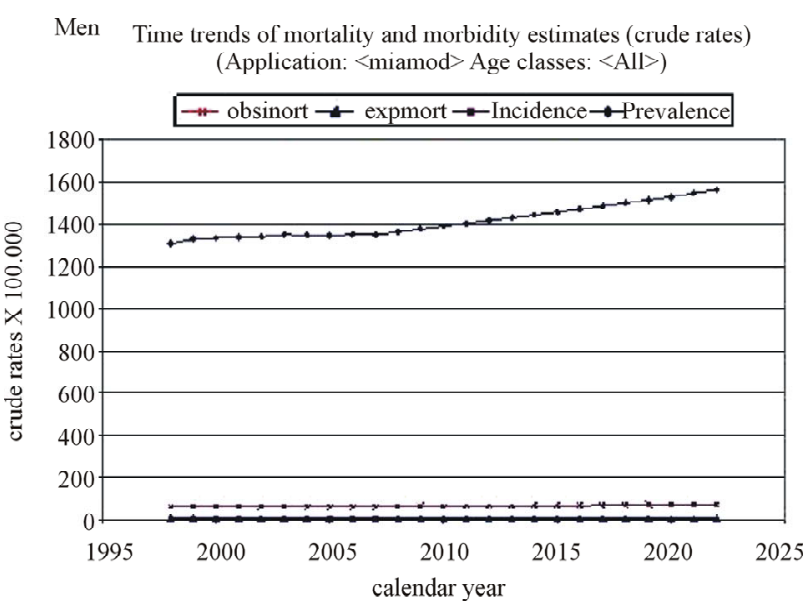

Figure 1. Time trends of mortality and morbidity estimates in men.

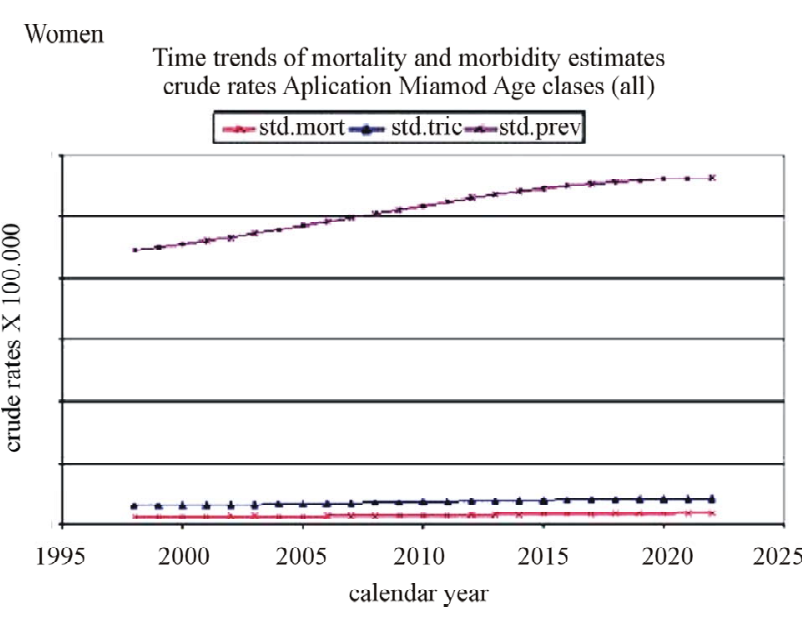

Figure 2. Time trends of mortality and morbidity estimates in women. 
Table 1. Projections the prevalence rates, incidence and mortality from melanoma cancer in men.

\begin{tabular}{|c|c|c|c|c|c|c|}
\hline Years & Prevalence & std. Prevalence & Incidence & std. Incidence & Mortality & std. Mortality \\
\hline 1998 & 73.72 & 52.31 & 11.85 & 8.52 & 5.84 & 4.12 \\
\hline 1999 & 75.92 & 52.78 & 12.13 & 8.57 & 5.97 & 4.12 \\
\hline 2000 & 76.72 & 53.24 & 12.2 & 8.61 & 5.96 & 4.11 \\
\hline 2001 & 77.64 & 53.61 & 12.26 & 8.63 & 5.97 & 4.1 \\
\hline 2002 & 78.09 & 54.02 & 12.29 & 8.66 & 5.98 & 4.12 \\
\hline 2003 & 79.41 & 54.44 & 12.42 & 8.7 & 6.05 & 4.14 \\
\hline 2004 & 79.44 & 54.78 & 12.38 & 8.74 & 6.03 & 4.15 \\
\hline 2005 & 79.74 & 55.27 & 12.38 & 8.79 & 6.02 & 4.18 \\
\hline 2006 & 80.23 & 55.5 & 12.42 & 8.81 & 6.05 & 4.19 \\
\hline 2007 & 80.1 & 55.83 & 12.38 & 8.84 & 6.03 & 4.21 \\
\hline 2008 & 81.19 & 56.1 & 12.52 & 8.88 & 6.1 & 4.23 \\
\hline 2009 & 82.16 & 56.42 & 12.66 & 8.92 & 6.16 & 4.25 \\
\hline 2010 & 83.07 & 56.84 & 12.79 & 8.97 & 6.23 & 4.27 \\
\hline 2011 & 84.02 & 57.07 & 12.93 & 8.99 & 6.29 & 4.29 \\
\hline 2012 & 84.97 & 57.39 & 13.07 & 9.03 & 6.35 & 4.3 \\
\hline 2013 & 85.81 & 57.6 & 13.2 & 9.07 & 6.4 & 4.31 \\
\hline 2014 & 86.66 & 57.88 & 13.34 & 9.1 & 6.45 & 4.32 \\
\hline 2015 & 87.53 & 58.26 & 13.47 & 9.15 & 6.53 & 4.35 \\
\hline 2016 & 88.42 & 58.48 & 13.62 & 9.18 & 6.6 & 4.37 \\
\hline 2017 & 89.31 & 58.78 & 13.77 & 9.23 & 6.67 & 4.39 \\
\hline 2018 & 90.27 & 58.96 & 13.94 & 9.26 & 6.76 & 4.41 \\
\hline 2019 & 91.28 & 59.16 & 14.11 & 9.3 & 6.85 & 4.43 \\
\hline 2020 & 92.52 & 59.46 & 14.32 & 9.34 & 6.95 & 4.45 \\
\hline 2021 & 93.96 & 59.74 & 14.55 & 9.38 & 7.07 & 4.47 \\
\hline 2022 & 95.46 & 60.02 & 14.79 & 9.43 & 7.19 & 4.49 \\
\hline
\end{tabular}

\subsection{General Features}

The method used is based on an estimation of the incidence, prevalence and mortality rates obtained from the best model that takes the age range-cohort effect into account simultaneously. The different methods available for calculating projections for non-transmissible diseases [11] are only valid provided they assume that the risk factors are equal in the period studied but may vary subsequently. A comparison of these methods [12] shows that they differ in terms of the risk factors employed. In the method used here, the fit of the observed mortality data was appropriate for both sexes. The melanoma mortality and incidence values for the period 1998-2000 estimated retrospectively using the MIAMOD program were similar to those from the Spanish registries con- sulted [13] and the most recent research in the field of cancer [14], thus implying that the estimated melanoma rates are comparable.

\subsection{Projections of Mortality Rates}

The mortality rates estimated in this study are slightly higher than those found in other Spanish melanoma studies [15] but similar to those provided by other European registries [16]. The finding that mortality rates are slowly decreasing also appears to be widespread, with some studies suggesting this is due to a lower mortality in older age groups [17]. Although this trend is currently being seen in Europe, the projections for the two sexes differ. Thus, the projections up until 2025 in the UK suggest a slight increase in mortality in men but not in 
Table 2. Projections the prevalence rates, incidence and mortality from melanoma cancer in women.

\begin{tabular}{|c|c|c|c|c|c|c|}
\hline Years & Prevalence & std. Prevalence & Incidence & std. Incidence & Mortality & std. Mortality \\
\hline 1998 & 54.04 & 44.54 & 3.79 & 3.04 & 1.67 & 1.24 \\
\hline 1999 & 54.84 & 45.00 & 3.85 & 3.07 & 1.69 & 1.24 \\
\hline 2000 & 55.37 & 45.51 & 3.88 & 3.11 & 1.68 & 1.24 \\
\hline 2001 & 56.56 & 46.12 & 3.95 & 3.15 & 1.7 & 1.25 \\
\hline 2002 & 56.82 & 46.67 & 3.97 & 3.2 & 1.68 & 1.25 \\
\hline 2003 & 57.64 & 47.29 & 4.03 & 3.25 & 1.69 & 1.27 \\
\hline 2004 & 58.36 & 47.91 & 4.08 & 3.3 & 1.72 & 1.29 \\
\hline 2005 & 59.21 & 48.58 & 4.14 & 3.36 & 1.74 & 1.32 \\
\hline 2006 & 60.07 & 49.23 & 4.21 & 3.42 & 1.78 & 1.35 \\
\hline 2007 & 60.86 & 49.88 & 4.28 & 3.48 & 1.81 & 1.38 \\
\hline 2008 & 62.16 & 50.50 & 4.39 & 3.54 & 1.86 & 1.41 \\
\hline 2009 & 63.51 & 51.15 & 4.51 & 3.6 & 1.92 & 1.45 \\
\hline 2010 & 64.92 & 51.79 & 4.63 & 3.66 & 1.98 & 1.48 \\
\hline 2011 & 66.4 & 52.42 & 4.76 & 3.72 & 2.05 & 1.52 \\
\hline 2012 & 67.91 & 53.01 & 4.89 & 3.79 & 2.11 & 1.55 \\
\hline 2013 & 69.47 & 53.53 & 5.04 & 3.85 & 2.18 & 1.59 \\
\hline 2014 & 71.06 & 54.05 & 5.18 & 3.9 & 2.26 & 1.62 \\
\hline 2015 & 72.69 & 54.52 & 5.33 & 3.96 & 2.34 & 1.66 \\
\hline 2016 & 74.34 & 54.97 & 5.49 & 4.01 & 2.42 & 1.69 \\
\hline 2017 & 76.05 & 55.34 & 5.64 & 4.06 & 2.51 & 1.72 \\
\hline 2018 & 77.75 & 55.61 & 5.8 & 4.1 & 2.6 & 1.75 \\
\hline 2019 & 79.48 & 55.86 & 5.97 & 4.13 & 2.69 & 1.78 \\
\hline 2020 & 81.25 & 56.03 & 6.13 & 4.17 & 2.78 & 1.81 \\
\hline 2021 & 83.04 & 56.17 & 6.29 & 4.19 & 2.88 & 1.84 \\
\hline 2022 & 84.81 & 56.18 & 6.46 & 4.21 & 2.98 & 1.86 \\
\hline
\end{tabular}

women [18]. However, we have found that the mortality increase is only statistically significant in women, with an annual percentage change of $2 \%$ until 2022, as according to the data collected by the Spanish Cancer Registry for 2001, the adjusted mortality rates are lower [19].

Trend studies in the USA [20] have shown a mortality decrease of $0.6 \%$ in women for the period 1989-2007 but an increase of $0.2 \%$ in men over the same period.

As regards the APC in Europe [21], it has been found that the mortality in countries such as Austria $(1.87 \%$ in men and $1.68 \%$ in women), Finland $(3.55 \%$ and $2.77 \%$, respectively) and Italy (5.83\% and $5.57 \%$, respectively) increased up until 2001. The values for Brazil $(4.20 \%$ and $4.68 \%$, respectively) were similar to those for Spain.

\subsection{Projections of Incident Rates}

Different incident rates have been reported depending on the geographic region studied and between sexes.

In the opinion of several authors, the incidence in men is continually increasing [22], although it should be noted that the annual percentage changes in those countries for which more recent data are available are lower than those for previous years. Thus, in the USA for example [23], the APC for the period 1975-1996 was $42.2 \%$ for men and $5.0 \%$ for women, whereas the increase over the period 1996-2007 was much lower $(2.0 \%$ and $2.3 \%$, respectively), thus suggesting a reduced incidence.

A similar situation is found as regards the different in- 
cident rates observed recently in European countries [24], where the APC is always lower in women and a slowing down or levelling out of the annual increase is observed for both sexes. Real decreases in melanoma incidence have only been observed in Hong Kong in a study performed up to 2002 [25].

In the projections estimated in this work, the incidence rate in men continues to increase but that in women begins to slowly decrease, although this trend is not statistically significant $(\mathrm{p}=0.89)$.

Differences between race and gender affect the onset of melanoma. Data for the USA in 2007 [26] show that melanomas are 29-times more prevalent in Caucasian men, and 19-times more prevalent in Caucasian women, than in their Afro-American counterparts.

In our setting, we have observed that the risk of melanoma begins to decrease in men after the year 2000 birth cohort (Figure 3) - in women it begins to decrease a decade earlier - a finding that has also been observed in other countries [27]. However, the projections study shows that mortality, but not incidence, is expected to increase at a rate of $2 \%$ per year in women (CI 1.90; 2.11). Neither incidence nor mortality is expected to increase in men to any statistically significant extent (Table 3).

Although a number of awareness campaigns have been undertaken in Spain, we believe that these need to be continued as the incidence rates for this disease continue to increase, although more slowly than previously. Furthermore, secondary prevention has been undertaken in a systematic manner and any type of nevus detected on the
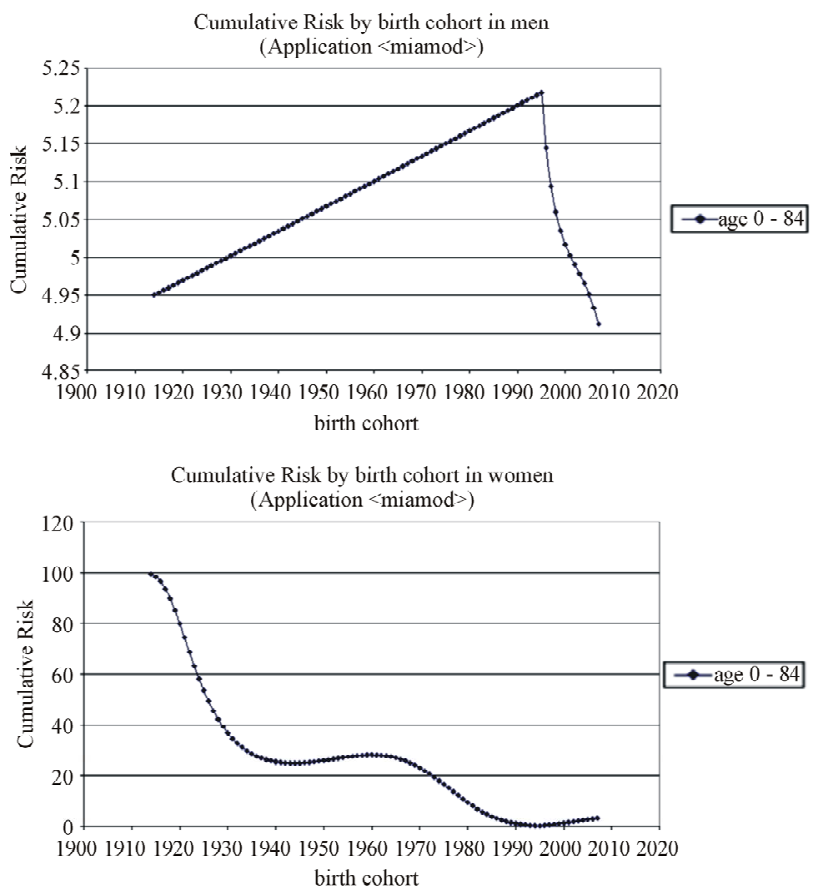

Figure 3. Cumulative Risk by birth cohort in men and women.
Table 3. Annual Percent Change (APC) between 2008 and 2022.

\begin{tabular}{ccccc}
\hline Rates & Sex & years & APC & IC 95\% \\
\hline Incidence & women & $2008-2022$ & 4.7 & $(-7.73,18.7)$ \\
Mortality & women & $2008-2022$ & 2.0 & $(1.9,2.1)$ \\
Incidence & men & $2008-2022$ & -2.0 & $(-12.0,9.1)$ \\
Mortality & men & $2008-2022$ & 2.3 & $(-4.8,11.31)$ \\
\hline
\end{tabular}

PAC: Annual Percent Change (APC).

skin has been controlled at both a specialist and GP level. These aspects, together with recent improvements in treatment [28], may have altered the behaviour of the population. However, the evolution of this disease must continue to be monitored as a key part of both primary and secondary prevention in order to decrease both the incidence and mortality of this preventable disease.

\section{REFERENCES}

[1] D. M. Parkin, S. L. Whelan, J. Ferlay, L. Teppo and D. B. Thomas, "Melanoma in: Cancer in Five Continents," No. 155, IARC Scientific Publication. http://www.iarc.fr/en/publications/pdfs-online/epi/sp155/ CI5V8.pdf" $\backslash \mathrm{t}$ "_blank

[2] Centro Nacional de Epidemiologia. http://cne.isciii.es/

[3] Instituto Nacional de Estadistica (INE). hptt// www.ine.es

[4] G. De Angelis, R. de Angelis, L. Frova and A. Verdecchia, "MIAMOD: A Computer Package to Estimate Chronic Disease Morbidity Using Mortality and Survival Data," Computer Methods and Programs in Biomedicine, Vol. 44, No. 2, 1994, pp. 99-107. doi:10.1016/0169-2607(94)90091-4

[5] W. Weibull, "A Statistical Distribution Function of Wide Applicability," Journal of Applied Mechanics, Vol. 18, 1951, pp. 293-297.

[6] http:// www.istitutotumori.mi.it

[7] http://www.ine.es/jaxi/tabla.do?path=/t15/p417/a2009/10/ $\&$ file $=02003 . p x \&$ type $=$ pcaxis $\& \mathrm{~L}=0$

[8] Joinpoint Program. http://srab.cancer.gov/joinpoint/

[9] N. Nasser, "UVB: Susceptibility in Malignant Melanoma," Anais Brasileiros de Dermatologia, Vol. 85, No. 6, 2010, pp. 843-848. doi:10.1590/S0365-05962010000600010

[10] T. Shibata, A. Kokubu, M. Miyamoto, Y. Sasajima and N. Yamazaki, "Mutant IDH1 Confers an in Vivo Growth in a Melanoma Cell Line with BRAF Mutation," American Journal of Pathology, Vol. 178, No. 3, 2011, pp. 13951402. doi:10.1016/j.ajpath.2010.12.011

[11] Nordpred, "A Software for Predicting Trends in Cancer Incidence". http://www.kreftregisteret.no/en/Research/

[12] T. Dyba and T. Hakulinen, "Comparison of Different Approaches to Incidence Prediction Based on Simple Interpolation Techniques," Statistics in Medicine, Vol. 19, No. 
13, 2000, pp. 1741-1752.

doi:10.1002/1097-0258(20000715)19:13<1741::AID-SIM 496>3.0.CO;2-O

[13] http://www-dep.iarc.fr/WHOdb/table3.asp

[14] M. P. Curado, B. Edwards, H. R. Shin, et al., "Cancer in Five Incidence," No. 160, IARC Scientific Publication, Lyon, 2009. http://ci5.iarc.fr/

[15] R. Marcos-Gragera, N. Vilar-Coromina, J. Galceran, J. Borràs, R. Clèries, J. Ribes, R. Gispert, A. Izquierdo and J. M. Borràs, "Rising Trends in Incidence of Cutaneous Malignant Melanoma and Their Future Projections in Catalonia, Spain: Increasing Impact or Future Epidemic?” Journal of the European Academy of Dermatology and Venereology, Vol. 24, No. 9, 2010, pp. 1083-1088.

[16] E. de Vries and J. W. Coebergh, "Cutaneous Malignant Melanoma in Europe," European Journal of Cancer, Vol. 40, No. 16, 2004, pp. 2355-2366. doi:10.1016/j.ejca.2004.06.003

[17] A. Stang and K. H. Jöckel, "Declining Mortality Rates for Nonmelanoma Skin Cancers in West Germany, 1968-99," British Journal of Dermatology, Vol. 150, No. 3, 2004, pp. 517-522.

[18] A. H. Olsen, D. M. Parkin and P. Sasieni, "Cancer Mortality in the United Kingdom: Projections to the Year 2025," British Journal of Cancer, Vol. 99, No. 9, 2008, pp. 1549-1554. doi:10.1038/sj.bjc.6604710

[19] G. de España, "Registro de Câncer de España. Incidencia y Mortalidad por Cancer 2001-2005," 2011. http://saludpublicaEspaña.es

[20] SEER. http://seer.cancer.gov/statfacts/html/breast.html
[21] http://www.who.int/healthinfo/statistics/mortality_rawdat a/en/index.html

[22] M. Pollan and G. Lopez-Abente, "Mortality Trends in Cutaneous Malignant Melanoma in Spain 1967-1986," Cancer Epidemiology, Biomarkers \& Prevention, Vol. 2, No. 6, 1993, pp. 545-550.

[23] S. F. Altekruse, C. L. Kosary, M. Krapcho, N. Neyman, R. Aminou, W. Waldron and B. K. Edwards, "SEER Cancer Statistics Review, 1975-2007,” 2010.

[24] A. V. Giblin and J. M. Thomas, "Incidence, Mortality and Survival in Cutaneous Melanoma," Journal of Plastic, Reconstructive \& Aesthetic Surgery, Vol. 60, No. 1, 2007, pp. 32-40. doi:10.1016/j.bjps.2006.05.008

[25] M. Makredes, S. K. Hui and A. B. Kimball, "Melanoma in Hong Kong between 1983 and 2002: A Decreasing Trend in Incidence Observed in a Complex Socio-Political and Economic Setting," Melanoma Research, Vol. 20, No. 5, 2010, pp. 427-430.

[26] http://seer.cancer.gov/statfacts/html/melan.html

[27] B. L. Diffey, "The Future Incidence of Cutaneous Melanoma within UK," British Journal of Dermatology, Vol. 151, No. 4, 2004, pp. 868-872. doi:10.1111/j.1365-2133.2004.06216.x

[28] A. C. Geller, S. M. Swetter, K. Brooks, M. F. Demierre and A. L. Yaroch, "Screening, Early Detection, and Trends for Melanoma: Current Status (2000-2006) and Future Directions," Journal of the American Academy of Dermatology, Vol. 57, No. 4, 2007, pp. 555-572. doi:10.1016/j.jaad.2007.06.032 